\section{Contemporary perspective on the use of fluoride products in caries prevention}

\author{
J. M. ten Cate $^{1}$
}

VERIFIABLE CPD PAPER

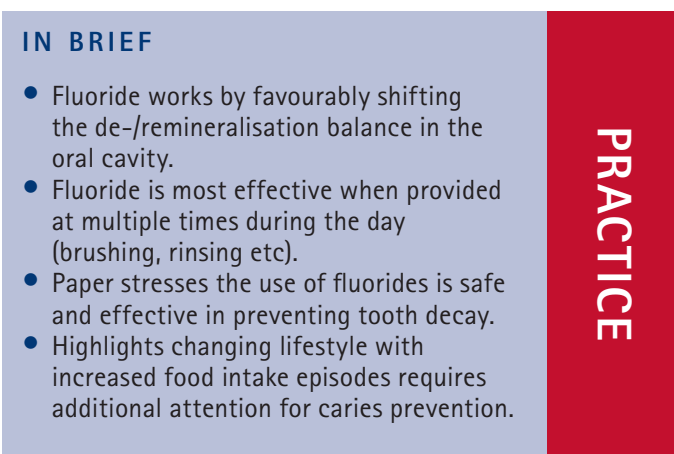

\begin{abstract}
Dental caries has declined in the 40 years since fluoridated toothpastes were introduced. Much has been learned about why fluoride is so effective and how this knowledge can be used to optimise programmes for caries prevention. Fluoride works through enhancing the remineralisation of early stages of caries and by inhibiting demineralisation, which would lead to dental caries. Remineralisation involves the deposition of calcium phosphates from saliva to rebuild partly dissolved enamel crystallites. When fluoride is incorporated the dissolution of these reinforced crystallites will be reduced during a subsequent sugar-induced and bacteria-mediated acid attack. Fluoride works primarily when it is present in the oral cavity. Based on our understanding of the fluoride mode of action the following advice can be given from clinicians to their patients: The fluoride concentration in oral products is related to efficacy but the concentration does not necessarily need to be high to be efficacious. Fluoride availability throughout the day is important; this can be achieved when fluoride products are used as part of the daily hygiene routine (F-brushing or rinsing). Alternatively, when fluoride is provided in the drinking water or through professionally applied F-varnishes or gels, the patient will benefit without requiring daily compliance to its use. The latter methods are particularly effective as additional treatments in high caries individuals.
\end{abstract}

\section{INTRODUCTION}

In the past decades fluoride toothpastes, rinses and other forms of fluoride delivery have become well established and these products are now being used by the majority of our patients. Many discoveries have deepened our understanding of the mode of action of fluoride in caries prevention. This information has resulted in a more rational use of fluorides and tailored advice for specific patient groups. Although a lot has been achieved with fluoridated toothpastes, there is still room for improvement regarding the effective delivery of fluoride in other vehicles. It is the aim of this article to describe the knowledge currently available on this topic and to suggest how this information could be used by clinicians to advise their patients on the ideal use of the currently available products. This article

\footnotetext{
'Department of Preventive Dentistry, Academic Center for Dentistry Amsterdam (ACTA), University of Amsterdam and Free University of Amsterdam, Gustav Mahlerlaan 3004, 1081 LA Amsterdam, the Netherlands Correspondence to: Professor J. M. ten Cate Email: j.t.cate@acta.nl; Tel: +31 205980440
}

\section{Refereed Paper}

Accepted 8 November 2012

DOI: 10.1038/sj.bdj.2013.162

${ }^{\circledR}$ British Dental Journal 2013; 214: 161-167 is essentially limited to fluoride products that can be used by patients and does not include a description of fluoridation of the drinking water, professionally applied fluoride products or fluoridation schemes that have limited, very local use, such as fluoridation of salt or milk.

\section{EPIDEMIOLOGY OF DENTAL CARIES, WHAT HAVE WE LEARNED FROM THE PAST?}

Throughout history dental caries has been a disease associated with wealth, as wealth meant the possibility to buy and consume luxury food. These were often rich in fermentable carbohydrates (sugars) and sugars were therefore considered the 'arch criminals' of the dentition. Dental caries is monitored by various parameters, such as the number of caries-free individuals at a given age or the number of decayed, filled or extracted teeth. The latter DMFT index (decayed missing filled teeth) is also reported at the level of the individual tooth surfaces and then referred to as DMFS. In the pre-fluoride era, DMFT values of 15-year-olds typically averaged around $10{ }^{1}$ This value fell sharply after the widespread use of fluoride products, initially in particular of fluoride toothpastes. The World Health Organization set a goal for 2000 that a level of DMFT below one should be reached. This goal was reached or approached in many economically more developed countries, but not necessarily in economically less developed countries. ${ }^{2}$ In addition a general observation has been that not everyone in society has benefited to the same extent from caries preventive programmes. Currently most of the dental decay is found in the socioeconomically lowest quartile of society. From a disease that was pandemic in society and where patients were happy to be provided with a denture early in life to be relieved of tooth ache, caries is now a disease affecting specific groups of individuals.

Where epidemiologic data projected a bright future for oral health, recent studies point to a stagnation of this improvement and in several countries even a reversal. ${ }^{3}$ Caries increases were reported for countries that initially showed the biggest improvements, such as Norway. ${ }^{4}$ In the United Kingdom improvements were still found during the last decade, ${ }^{5,6}$ while in the United States oral health improved in adults but worsened in 2-5-year-olds. ${ }^{7}$ 
Given that information it is important to reconsider the established caries prevention schemes aiming to optimise their benefits. Any programme should be based on a comprehensive knowledge of the aetiology and pathogenesis of the disease and how preventative or curative medication may be effective.

\section{FLUORIDE - WHAT DO WE KNOW ABOUT ITS ROLE AND IMPOR- TANCE IN CARIES PREVENTION?}

The prevention of dental caries is uniquely associated with fluorides. Since the finding of fluorosis in patients with low levels of decay in the 1930s, and the elaborate study of the causing factor, fluoride has been studied intensely both clinically and in laboratory experiments. ${ }^{8,9}$ It took considerable efforts to formulate toothpastes containing fluoride into efficacious products. Various randomised clinical trials (RCTs) have shown that proper formulation of a toothpaste is critical to achieving maximum efficacy. The caries reduction achieved by the regular use of fluoride toothpastes is now generally accepted, it is confirmed in multiple RCTs and documented in the prestigious Cochrane library. ${ }^{10}$

Why is fluoride effective and what parameters determine the magnitude of its effect? To better understand this a small side step to describe why and how dental caries develops needs to be made.

\section{DENTAL CARIES - HOW IS IT FORMED?}

Dental caries is caused by the interplay of three factors: the presence of fermentable dietary sugars, bacteria present in dental plaque and obviously the availability of teeth. Numerous bacteria thrive in the oral cavity where they form a bacterial deposit onto the surfaces of the teeth. This dental plaque, now often called the dental biofilm, has properties similar to biofilms formed elsewhere in the body and in nature. ${ }^{11}$ Well-controlled biofilms serve many good purposes. ${ }^{12}$ However, when biofilms grow out of proportion on a tooth two processes may combine that can lead to tooth decay:

- Bacteria use sugars to produce the matrix of the biofilm, which helps the bacteria to adhere to the surface and shield them from outside influences

- Several bacteria metabolise sugars present in the diet and thereby form

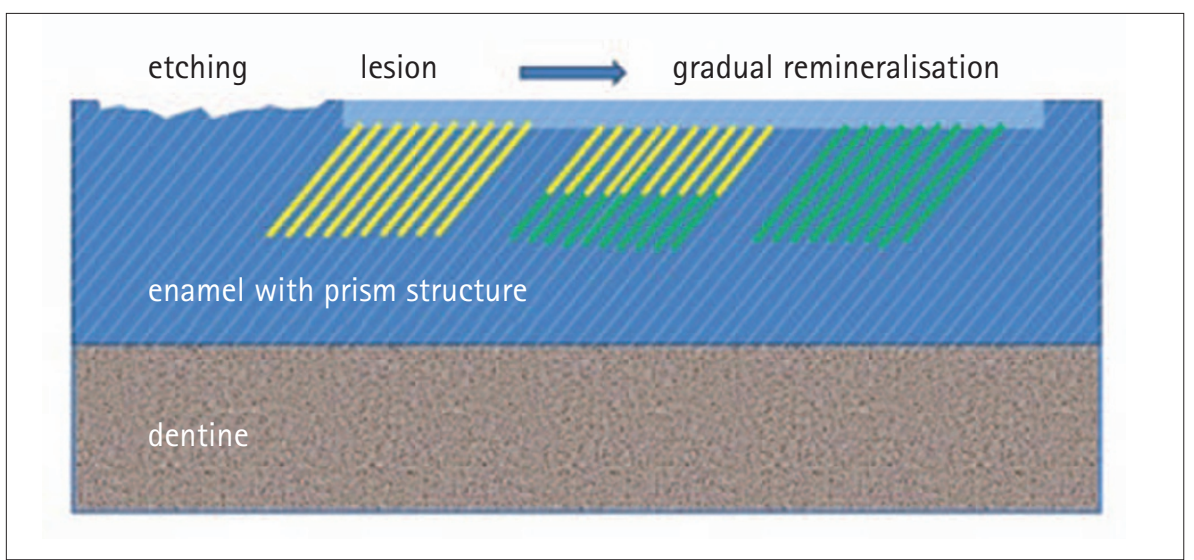

Fig. 1 Schematic of cross section of tooth depicting structural changes occurring during tooth erosion, and de- and remineralisation (from left to right). With erosion an integral layer of enamel is removed by strong acids; this layer is not restored after the $\mathrm{pH}$ is returned to neutrality. In contrast, weak organic acids formed in dental plaque, when sugars are metabolized, lead to preferential dissolution in the spaces between the enamel prisms. These acids bypass the fluoride rich outer enamel layer. When $\mathrm{pH}$ return to neutrality calcium and phosphate ions from saliva can precipitate in these dissolved regions. In the presence of fluoride this leads to an enforced mineral, less susceptible to future acid attacks.

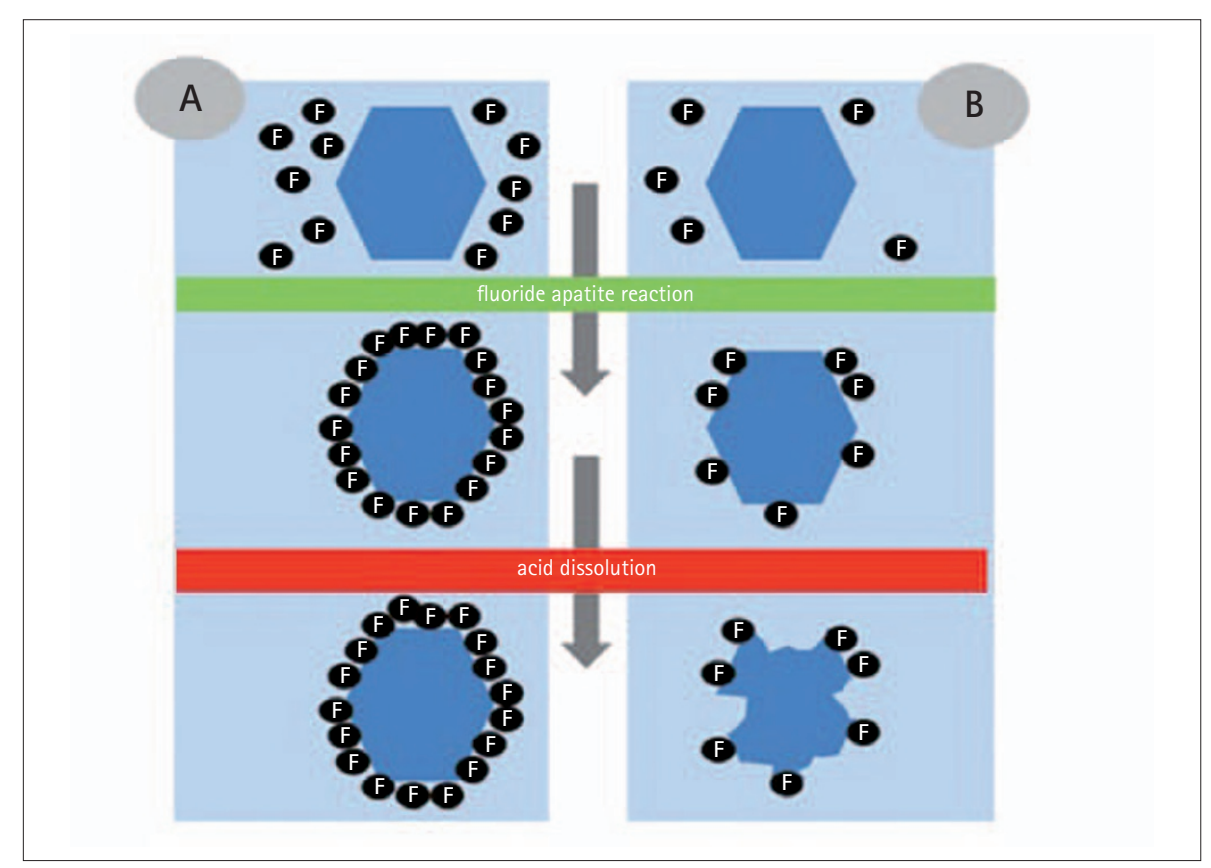

Fig. 2 Events taking place at the surface of hydroxyapatite crystalliyes. Fluoride-ions are adsorbed onto the crystals. Full coverage $(A)$ will prevent dissolution of the crystallites during an acid attack. Partial coverage (B) will lead to partial dissolution (modified from Arends and Christoffersen, ${ }^{32}$ and Buzalaf et $a .^{33}$ )

acids. During prolonged contact with these acids, the enamel or dentine in teeth will dissolve.

Like bone, teeth are formed from the calcium phosphate mineral hydroxyapatite (HAP). The solubility of HAP depends on the $\mathrm{pH}$ (acidity, alkalinity), but also on the ionic-levels of the HAP components (calcium and phosphate) of the surrounding medium. Under physiological conditions saliva and dental biofilms have a $\mathrm{pH}$ near neutrality ( $\mathrm{pH}$ 7). The oral fluids contain ionic calcium and phosphates and their levels together with the $\mathrm{pH}$ determine whether a condition of so-called superor under-saturation exists. In every day words this means whether a mineral will precipitate or dissolve. For the oral cavity this implies whether minerals form onto or inside the teeth, (as calculus or enamel crystallites) or, alternatively, enamel will dissolve. As mentioned above, sugars metabolised in oral biofilms form acids that dissolve hydroxyapatite.

Enamel is built from periodic 'prismatic' structures, each comprising millions of HAP crystallites. Together this creates a 


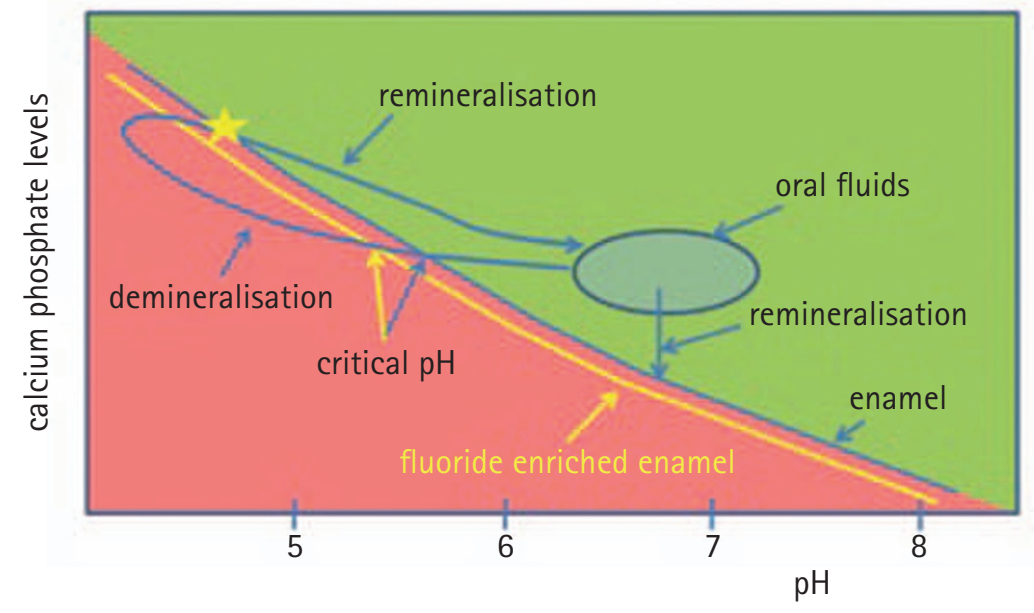

Fig. 3 Graph depicting the primary modes of action of fluoride: decreasing demineralisation and enhancing of remineralisation. Solubility of hydroxyapatite (HAP) depends on $\mathrm{pH}$ of the oral fluids (plaque). At physiological pH (6-7), the calcium and phosphate levels are supersaturated with respect to HAP and can lead to remineralisation (green region). When acids are formed in the plaque, the $\mathrm{pH}$ decreases and beyond the critical $\mathrm{pH}$ this leads to dissolution of HAP (red region). This leads to an increase of the calcium and phosphate levels. When all sugars are metabolised and $\mathrm{pH}$ is increased due to salivary buffering (and HAP dissolution) the return part of the cycle is started. At some stage (see star) mineral will start to reprecipitate. The presence of fluoride leads to lower solubility (see yellow line), hence a lower (critical) $\mathrm{pH}$ at which dissolution starts, and a lower $\mathrm{pH}$ at which mineral reprecipitation occurs

mechanically strong structure that from a chemical point of view is rather vulnerable. Due to porosities present in this structure, both between the enamel prisms and between the HAP crystallites, the 'weak' organic acids formed in the plaque will penetrate into enamel rather than dissolve the enamel layer by layer. In contrast, the latter occurs when enamel is etched by 'strong' acids, such as phosphoric acid. The latter etching is common practice in the dental surgery to roughen enamel to create micro-retention required for sealants or restorations. Penetration of acids will lead to selective dissolution inside the tooth, with the surface layer generally being bypassed as this is less soluble due to the accumulation of fluoride (Fig. 1). If this demineralisation process continues with thousands of acid challenges, the tooth gradually dissolves over an extended depth, while still maintaining overall integrity. However, when demineralisation extends to greater depth the overall structure might become too weak and the remaining tissue might collapse with a big cavity as a result! The described structural properties are not necessarily a shortcoming, as new enamel may be formed in the demineralisation defects ('white spots') by the deposition of calcium- and phosphateions from saliva, when the plaque $\mathrm{pH}$ has returned to neutrality. This natural repair mechanism is known as remineralisation. When fluoride ions are present during remineralisation, they become incorporated in the apatite structure, forming fluor-hydroxyapatite mixed crystals. As a result of the fluoride incorporation these newly formed or partly regrown crystallites have a lower solubility than the original ones. Moreover, in the presence of low fluoride levels this repair process is accelerated (Fig. 2). The 'caries attack, crystallite dissolution and repair' cycle will, in case of fluoride availability, result in repaired tooth enamel that is, in principle, slightly more resistant to future acid challenges (Fig. 3). However, the full story is more complicated, as will be described below.

\section{WHY IS FLUORIDE EFFECTIVE IN INHIBITING DENTAL CARIES WITH REGULAR EXPOSURE?}

Part of this question was already addressed in the preceding paragraph but the arsenal of fluoride's mode of action in caries prevention is more elaborate. Numerous studies, particularly in the 1970-90s have disclosed why fluoride is effective and how this information can be used to further develop products and concepts for caries prevention. The original understanding of the mode of action of fluoride was that if teeth would be loaded with fluoride, meaning fluoride incorporated into the crystalline structure, dental caries would not develop. This hypothesis was tested in a very ingenious experiment in which small pieces of dental enamel ('chips') were placed in dentures of volunteers who were asked to rinse with sucrose solutions to create highly cariogenic conditions. ${ }^{13}$ Obviously for ethical reasons this could not be done with volunteers still in possession of their natural dentition. After several weeks the chips were removed and analysed with advanced microscopic techniques enabling the visualisation of decay long before it could be seen clinically. The creative concept of this study was that not only human enamel was placed in the dentures, but also shark enamel which is composed of fluorapatite, and secondly that volunteers were, in some experimental periods, asked to rinse with a $0.2 \%$ sodium fluoride solution. With this experimental design the investigators could discriminate between the contribution of fluoride present in the enamel (in the shark enamel group), and the available of fluoride in the oral fluid, on the initiation of tooth decay. The outcome was such that while there was some protection from tooth bound 'intrinsic' fluoride, the extrinsically provided fluoride was considerably more effective in preventing decay. Consequently, fluoridation of the enamel, with the aim of producing high levels of incorporated fluoride, was proven not to be a sufficient method to inhibit tooth decay. Clinically this is confirmed in patients that stop using fluoride products and develop tooth decay. ${ }^{14}$

This and other studies confirmed that a frequent exposure to fluoride products is more beneficial in caries prevention than the incorporation of fluoride into the dental tissues, such as is achieved by semiannual topical fluoride treatments.

This paradigm shift paved the way for oral care products that lead to the elevation of the fluoride levels in the oral cavity, preferably several times during the day. It was found that this increase does not necessarily have to be substantial. Studies where fluoride-slow-release-devices were placed in the mouth of high caries children confirmed that a relatively small increase in fluoride levels in oral fluids (in these studies from $0.03 \mathrm{ppm}$ to $0.11 \mathrm{ppm}$ ) gave $73 \%$ reduction 
of caries scores in deciduous and 64\% caries reduction in the permanent dentition. ${ }^{15}$

The fluoride levels in the oral cavity are generally relatively low as fluoride is cleared from the mouth due to salivary secretion and swallowing. Therefore the effect of fluoride after using oral care products on bacteria is limited. Bacterial growth and metabolism are affected by fluoride concentrations exceeding about $10 \mathrm{ppm}$. Such fluoride levels are limited to a very short period after using a fluoride product. Fluoride containing products may be effective as antimicrobials but in those cases this is generally attributable to other components, such as the fluoride counter ions (amine, stannous), preservatives, surfactants or antimicrobials added specifically for that purpose (zinc salts, Triclosan, essential oil extracts, etc.). ${ }^{12}$ Regarding its mode of action in caries prevention the consensus today is that fluoride is mainly effective by enhancing the remineralisation of initial caries defects and by inhibiting the demineralisation that would lead to caries initiation or progression. Fluoride thus shifts the 'demin-remin balance' from net demineralisation, in the case of caries active patients, towards net remineralisation. ${ }^{9}$ It should be emphasised that fluoride is effective when present in the oral cavity and not after it has been swallowed.

\section{CAN FLUORIDE BE STORED IN THE ORAL CAVITY?}

Fluoride levels in saliva and biofilms decrease sharply with time after a topical application, rinsing or brushing with a fluoride dentifrice. Given this clearance the question is relevant how long the fluoride will be sufficiently elevated to benefit the de-/remineralisation processes. Data from a randomised clinical trial to evaluate dentifrices with various fluoride levels showed that even 18 hours after the last brushing, the fluoride concentrations in saliva and plaque reflected the dentifrice that had been used (Fig. 4). In saliva the fluoride concentration was increased from 0.01 to $0.02 \mathrm{ppm}$ and in plaque from 1.5 to $2.4 \mathrm{ng} \mathrm{F} / \mathrm{mg}$ wet weight for the 0 and $0.25 \%$ (2,500 ppm) fluoride dentifrice respectively. Also for the three products tested, containing $0.1 \%$ (1,000 ppm), $0.15 \%$ (1,500 ppm) and $0.25 \%(2,500 \mathrm{ppm})$ respectively, a dose response effect was found. These observations suggest the presence of fluoride reservoirs that are filled during the use of

Fluoride concentration in oral cavity

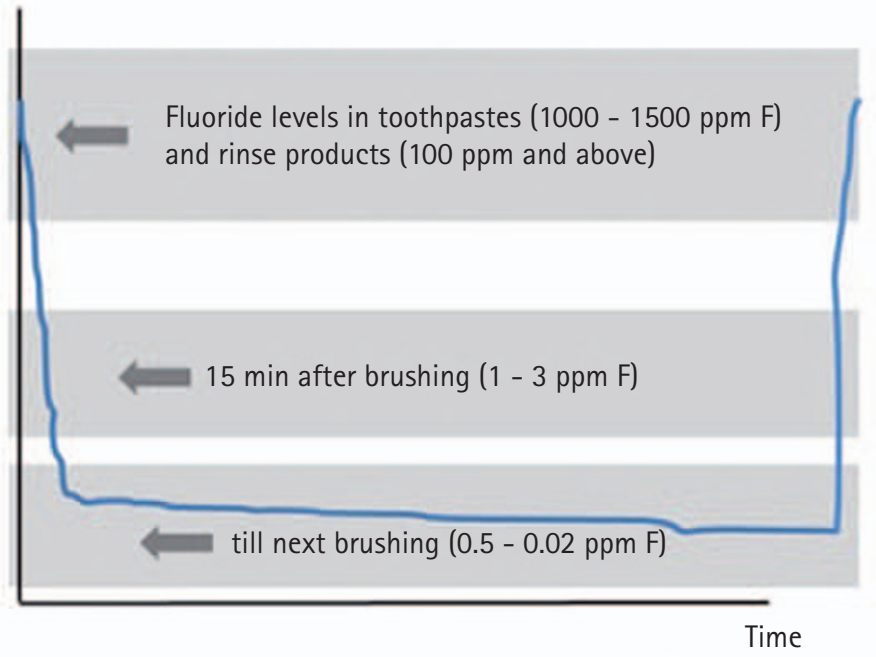

Fig. 4 Schematic of fluoride levels in the oral cavity after brushing or rinsing with a fluoride product. The fluoride concentration decreases sharply within minutes of using a product. The residual elevation still helps to promote remineralisation of the dental hard tissues

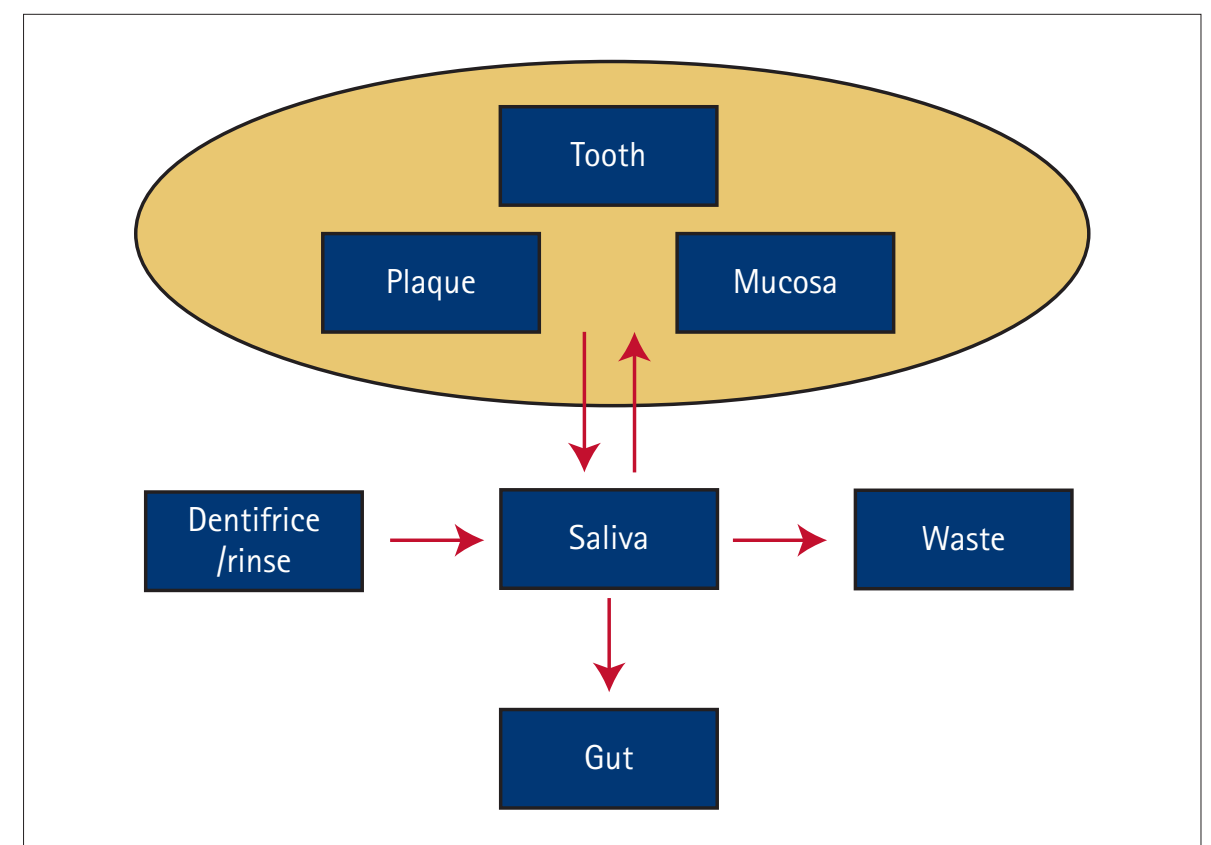

Fig. 5 The fate of fluoride after using a fluoride product. Fluoride in saliva is exchanged with plaque, mucosa and the tooth surface. In particular the mucosal surface provides a large depot serving for slow release of fluoride. Fluoride swallowed is partly stored in bones but not recycled back to the oral cavity (modified from Duckworth and Morgan ${ }^{16}$ )

a fluoride product and which will subsequently slowly release its fluoride. ${ }^{16}$ Fluoride is present in dental plaque, often associated with mineral components such as early calculus, but also adherent to the oral mucosa (Fig. 5). ${ }^{17}$ Particularly the mucosal surface is an underestimated fluoride retention site, which explains the high levels of salivary fluoride that can be achieved with fluoride rinses (100 ppm and above). ${ }^{18,19}$ Other fluoride depots in the oral cavity are calcium fluoride $\left(\mathrm{CaF}_{2}\right)$ precipitates which forms after topical fluoride applications, but not during toothbrushing. These $\mathrm{CaF}_{2}$ globules are protected from rapid dissolution by a phosphate-protein coating of salivary origin, which will open at low $\mathrm{pH}$, when incidentally the fluoride is most needed! Once again, the increase in bioavailable fluoride does not need to be substantial to have a favourable impact: even sub-ppm levels of fluoride have been shown to enhance remineralisation and inhibit demineralisation of enamel and dentine. ${ }^{9}$ 


\section{HOW DOES A FLUORIDE REGIME FIT IN THE PATIENT'S EVERY DAY LIFE?}

Summarising the above it may be concluded that effective caries preventive agents should be used daily. Various RCTs have reported that the level of caries prevention resulting from using a fluoride product is related to the frequency of its use. ${ }^{20}$ Additionally it was found that it is better to not completely rinse out the mouth with large amounts of water after using a product, since the fluoride left in the oral cavity after brushing contributes to the overall caries preventive effect. ${ }^{20,21}$ A recent review by experts concluded that one of the following methods would lead to increased post-brushing fluoride retention: (a) spit and don't rinse', (b) rinse with a slurry of toothpastes with saliva and (c) rinse with a mouth rinse containing fluoride. Each approach could be beneficial for caries control at the individual level, although high level evidence from randomised clinical trials is not yet available. ${ }^{18}$

Oral care products were shown to be particularly efficacious when their use was appreciated and accepted by the patient. Compliance is a major determining factor for the success of these products. The success of fluoridated toothpastes was probably driven by the societal changes starting in the 1970s when personal hygiene and aesthetics gained importance in society. However, the currently changing lifestyle in many patients is creating a new challenge for oral care products and for dental practitioners. The number of snacks and meals consumed per day has increased substantially, reason why the current eating pattern is often referred to as 'grazing' rather than eating. With many of these consumptions being rich in carbohydrates, hence cariogenic (and not to forget acidified sugary soft drinks or sweet alcoholic beverages, which are typically consumed over extended periods) it is doubtful if fluoride is sufficiently powerful to counteract the increased cariogenic challenge.

Another very different challenge for the dental community and manufacturers of oral care products is the constant flow of critical and negative publications pertaining to fluoride. Dental practitioners and personnel should be aware of this type of information that is available on public media and therefore very accessible to patients. If, for whatever reason, patients stop or reduce the use of fluoride products, there is no question that this will lead to detrimental effects on the individual's dentition. If a patient in your surgery shows a suddenly worsened dental situation, the possibility of a change in the used oral care products should be one of the first questions being asked.

A third risk factor in caries prevention is the latency of parents who have benefited from the use of fluoridated toothpastes but who are - not knowing the evil of serious decay - possibly less determined in monitoring brushing and snacking habits of their children. As an example, while cariogenic snacks were an official no-go to treat classmates in schools in the 1970-80s, this seems to no longer be a rule in many schools.

\section{HOW IS FLUORIDE DELIVERED IN THE ORAL CAVITY?}

Fluoridated toothpastes are, without question, the corner stone of caries prevention and largely responsible for its success. On average more than 95\% of the population in economically more developed countries use a fluoridated toothpaste. The maximum allowed fluoride level in toothpastes in Europe is $0.15 \%(1,500 \mathrm{ppm})$, which is higher than the maximum content for toothpastes in the USA, being $0.11 \%(1,100 \mathrm{ppm})$. The acceptance, widespread use and proven efficacy of fluoridated toothpastes have encouraged manufacturers to also include other ingredients into toothpaste. These multifunctional toothpastes are aimed at preventing or curing a wide range of oral conditions besides tooth decay, such as periodontal infections, halitosis, dentinal hypersensitivity, staining and erosion. In the past decades fluoride toothpastes have been developed, with $0.28 \%$ (2,800 ppm) and $0.5 \%$ (5,000 ppm) fluoride. These are available on prescription or as over-thecounter product, depending on the country. Fluoride toothpastes at 5,000 ppm were shown to be efficacious against root surface caries. ${ }^{22}$ Use of a 5,000 ppm F-paste gave significant additional caries reduction over a 1,450 ppm F-paste, and the high fluoride paste was particularly effective in subjects with irregular/ less frequent brushing habits. ${ }^{23} \mathrm{~A}$ high fluoride toothpaste might be suitable for patients with high caries challenges due to a cariogenic lifestyle. ${ }^{24}$ Although increasing the fluoride dose is a possibility, an advice to increase the frequency of brushing or other exposure such as rinsing is preferred. Obviously a more general advice, also in view of general health, would be to try to convert to a more healthy lifestyle.

Besides in toothpaste, fluoride has been studied when added to salt, milk, chewing gum and when supplied in rinses (discussed below) or tablets (for review see TubertJeannin et al. ${ }^{25}$ ). One of the benefits of such products is that they can also be used to bring fluoride into the mouth at times other than tooth brushing. Moberg Sköld et $a .^{26}$ studied additional fluoride rinses and varnishes when given supervised in a school environment and observed that even in groups of adolescents with moderate caries experience, caries initiation and progression were significantly reduced. An extensive Cochrane review, in which all published randomised clinical trial (RCT) data were combined, concluded that fluoride toothpastes, mouthrinses, gels and varnishes have a similar effectiveness for preventing caries. In addition this meta-review confirmed that additional caries reduction is achieved when other fluoride sources are combined with fluoride toothpaste. ${ }^{27}$ A summary of RCTs on fluoride concentration in toothpastes showed a positive dose response: pastes with 1,000-1,500 ppm $\mathrm{F}$ gave 23\% caries reduction compared to fluoride free placebo, this value increased to 36\% for pastes at around 2,500 ppm. For pastes below 1,000 ppm no significant difference with placebo was found, probably due to the small number of studies. The advice was that the decision on which fluoride (level) toothpastes to be used by children under six years of age should be made balanced with the risk of fluorosis. Hence this should be directed by factors like fluoride levels in the water supplies and other types of fluorides used.

\section{WHY SHOULD FLUORIDATED MOUTHRINSES BE USED?}

More data on the efficacy of fluoride products are available from studies where only fluoride levels in saliva and plaque were 
measured. While this is only a so called surrogate outcome measure it serves as a good indication how effective a particular regimen could be. Zero and colleagues performed various studies to compare fluoride and placebo toothpastes and rinses and reported that fluoride rinsing was in fact superior to fluoride tooth brushing, judging from the residual fluoride levels in both saliva and plaque. ${ }^{28}$ Obviously tooth brushing will remain necessary, irrespective of how poorly it is performed by the average patient in terms of plaque removal, as it is a habit that generally has a good compliance and delivers fluoride to the oral cavity. In studies with a similar design Duckworth et al. ${ }^{29}$ showed that rinsing with a fluoride free rinse decreased the fluoride levels in saliva. The preferred order of events would therefore be first tooth brushing, then additional cleaning of interdental spaces with floss or sticks followed by a fluoride rinse. Van Strijp et al. ${ }^{30}$ studied the benefits of an additional fluoride 'moment', using a fluoride rinse in addition to twice daily fluoride brushing. Such a routine was shown to benefit lesion remineralisation. An additional argument for using fluoride rinses to supplement the daily fluoride need is for patients that use electric toothbrushes, as they typically use a rather small quantity of toothpaste on the small brushes. Although not shown conclusively this might negatively impact the overall fluoride availability and hamper caries prevention.

A special case for fluoride rinses could be made for elderly patients. Often they take medication that affects salivary flow or they have a systemically impaired salivary function. Moreover, tooth brushing may be physically difficult due to loss of dexterity, strenuous for the patients themselves or too time consuming, when done carefully, for those taking care of their hygiene. Toothpastes could also be too aggressive for sensitive mucosal tissue, in particular when salivary function is reduced. Rinsing with an effective fluoride rinse that lacks aggressive additional components, would then be a valuable method to provide sufficient amounts of fluoride throughout the day. One should remember that this will never be a full replacement for tooth brushing, considering the importance of tooth brushing for periodontal health.

\section{ARE ALL FLUORIDES THE SAME?}

Fluoride in toothpastes comes in various chemical forms, as sodium fluoride, stannous fluoride, amine fluoride, monofluorophosphate etc. The choice by manufacturers in the past was often related to compatibility with abrasive elements, price of components etc. Although differences in anti-caries performance were observed in standard RCTs, ${ }^{31}$ the conclusion is justified that these differences are small compared to patient variables such as brushing time, frequency and post-brushing behaviour.

\section{CONCLUDING REMARKS}

After the beneficial effects of fluorides in caries prevention were found in the 1930s major improvements have occurred in our understanding of the fluoride mode of action and this has resulted in new products. Currently we can choose from a range of fluoride toothpastes, rinses, gels and varnishes that have proven clinical efficacy. Fluoridated toothpastes are, by far, the most frequently sold and used method of caries prevention. Given the fast clearance of fluoride after tooth brushing, the preferred order for fluoride delivery should, in my view, be brushing with a fluoridated toothpaste, additional cleaning of interdental spaces followed by using a fluoride rinse. The effectiveness of brushing and rinsing is very dependent on the patient's compliance.

In patients where compliance is hard to achieve fluoride schemes should be chosen that are efficacious without the cooperation of the patient. Fluoridation of the drinking water is still the optimal method of fluoride delivery but in many parts of the world this is not implemented due to political/emotional reasons. Alternatively, in high risk or non-compliant patients high fluoride gel and varnish treatments could be applied professionally.

The author has received a fee from Johnson $\mathcal{E}$ Johnson (JCLJ) for writing this review and has in the past acted as a consultant to and received research grants from several oral care companies.

1. Burton V J, Rob M I, Craig G G, Lawson J S. Changes in the caries experience of 12-year-old Sydney schoolchildren between 1963 and 1982. Med J Aust 1984; 140: 405-407.

2. Gao X L, McGrath C, Lin H C. Oral health status of rural-urban migrant children in South China. Int J Paediatr Dent 2011; 21: 58-67.

3. Bagramian R A, Garcia-Godoy F, Volpe A R. The global increase in dental caries. A pending public health crisis. Am J Dent 2009; 22: 3-8.

4. Haugejorden O, Birkeland J M. Analysis of the ups and downs of caries experience among Norwegian children aged five years between 1997 and 2003.
Acta Odontol Scand 2005; 63: 115-122.

5. Steele J, O'Sullivan I. Executive summary: Adult Dental Health Survey 2009. London: The Health and Social Care Information centre, 2011.

6. Drugan C S, Downer M C. Dental health in the United Kingdom and influencing variables. Bundesgesundheitsblatt Gesundheitsforschung Gesundheitsschutz 2011; 54: 1027-1034.

7. Dye B A, Tan S, Smith V et al. Trends in oral health status: United States, 1988-1994 and 1999-2004. Vital Health Stat 11 2007; 248: 1-92.

8. ten Cate J M. In vitro studies on the effects of fluoride on de-and remineralization. J Dent Res 1990; 69: 614-619; discussion 634-636

9. ten Cate J M, Featherstone J D. Mechanistic aspects of the interactions between fluoride and denta enamel. Crit Rev Oral Biol Med 1991; 2: 283-296.

10. Marinho V C, Higgins J P, Sheiham A, Logan S. Combinations of topical fluoride (toothpastes, mouthrinses, gels, varnishes) versus single topical fluoride for preventing dental caries in children and adolescents. Cochrane Database Syst Rev 2004 1: CD002781.

11. ten Cate J M. Biofilms, a new approach to the microbiology of dental plaque. Odontology 2006; 94: 1-9.

12. Marsh P D. Contemporary perspective on plaque control. Br Dent J 2012; 212: 601-606.

13. Ogaard B, Rølla G. The in vivo orthodontic banding model for vital teeth and the in situ orthodontic banding model for hard-tissue slabs. J Dent Res 1992; 71: 832-835.

14. Haugejorden $\mathrm{O}$, Lervik $T$, Birkeland J M, Jorkjend L. An 11-year follow-up study of dental caries after discontinuation of school-based fluoride programs. Acta Odontol Scand 1990; 48: 257-263.

15. Toumba K J, Curzon M E. A clinical trial of a slowreleasing fluoride device in children. Caries Res 2005; 39: 195-200.

16. Duckworth R M, Morgan S N. Oral fluoride retention after use of fluoride dentifrices. Caries Res 1991; 25: 123-129.

17. Duckworth $R$ M, Jones $Y$, Nicholson J, Jacobson A P, Chestnutt I G. Studies on plaque fluoride after use of F-containing dentifrices. Adv Dent Res 1994; 8: 202-207.

18. Pitts N, Duckworth R M, Marsh P, Mutti B, Parnell C, Zero D. Post-brushing rinsing for the control of dental caries: exploration of the available evidence to establish what advice we should give our patients. Br Dent J 2012; 212: 315-320.

19. Zero D T, Raubertas R F, Pedersen A M, Fu J, Hayes A L, Featherstone J D. Studies of fluoride retention by oral soft tissues after the application of homeuse topical fluorides. J Dent Res 1992; 71: 1546-1552.

20. Chesters R K, Huntington E, Burchell C K, Stephen $\mathrm{K}$ W. Effect of oral care habits on caries in adolescents. Caries Res 1992; 26: 299-304.

21. Sjögren K, Birkhed D, Rangmar S, Reinhold A C. Fluoride in the interdental area after two different post-brushing water rinsing procedures. Caries Res 1996; 30: 194-199.

22. Lynch $\mathrm{E}$, Baysan $\mathrm{A}$. Reversal of primary root caries using a dentifrice with a high fluoride content. Caries Res 2001; 35(Suppl 1): 60-64.

23. Nordström A, Birkhed D. Preventive effect of high-fluoride dentifrice $(5,000 \mathrm{ppm})$ in caries-active adolescents: a 2-year clinical trial. Caries Res 2010; 44: 323-331.

24. ten Cate J M, Buijs M J, Miller C C, Exterkate R A Elevated fluoride products enhance remineralization of advanced enamel lesions. J Dent Res 2008; 87: 943-947.

25. Tubert-Jeannin S, Auclair C, Amsallem E et al. Fluoride supplements (tablets, drops, lozenges or chewing gums) for preventing dental caries in children. Cochrane Database Syst Rev 2011; 12: CD007592.

26. Moberg Sköld U, Birkhed D, Borg E, Petersson L G. Approximal caries development in adolescents with low to moderate caries risk after different 3-year school-based supervised fluoride mouth rinsing programmes. Caries Res 2005; 39: 529-535.

28. Zero D T, Fu J, Espeland M A, Featherstone J D. 
Comparison of fluoride concentrations in unstimulated whole saliva following the use of a fluoride dentifrice and a fluoride rinse. J Dent Res 1988: 67: 1257-1262.

29. Duckworth R M, Horay C, Huntington E, Mehta V. Effects of flossing and rinsing with a fluoridated mouthwash after brushing with a fluoridated toothpaste on salivary fluoride clearance. Caries Res 2009; 43: 387-390.
29. Marinho V C. Cochrane reviews of randomized trials of fluoride therapies for preventing dental caries. Eur Arch Paediatr Dent 2009; 10: 183-191.

30. van Strijp A A, Buijs M J, ten Cate J M. In situ fluoride retention in enamel and dentine after the use of an amine fluoride dentifrice and amine fluoride/ sodium fluoride mouth rinse. Caries Res 1999; 33: $61-65$.

31. Stookey G K, DePaola P F, Featherstone J D et al. A critical review of the relative anticaries efficacy of sodium fluoride and sodium monofluorophosphate dentifrices. Caries Res 1993; 27: 337-360.

32. Arends J, Christoffersen J. Nature and role of loosely bound fluoride in dental caries. J Dent Res 1990; 69: 601-605, discussion 634-636.

33. Buzalaf M A, Pessan J P, Honório H M, ten Cate J M. Mechanisms of action of fluoride for caries control. Monogr Oral Sci 2011; 22: 97-114.

\section{Erratum}

Research summary (BDJ 2013; 214: 66-67)

Summary of: Developing professional status: an investigation into the working patterns, working relationships and vision for the future of UK clinical dental technicians

In the above research summary the abstract originally published was incorrect. The abstract should have read as follows:

Aims To investigate the working patterns and patient base of registered clinical dental technicians (CDTs); their relationships with dentists and other professionals in the dental team; their willingness to work within the NHS and their expectations for the future as a new professional group.Methods Face-to-face qualitative interviews of registered CDTs, selected because of their geographic representation and mode of working, informed the development of a postal questionnaire survey of all early registrants with the General Dental Council (GDC). Results The majority of CDTs reported working part-time, often combining clinical practice with their role as a dental technician. They reported both positive and negative working relationships with dentists and dental technicians, demonstrating collaboration and/or competition depending on whether the scope of CDTs was respected and patient care was shared or lost. CDTs role in the NHS was limited because they did not have the status of becoming a recognised provider of dental care. There was a desire to expand their scope of practice in future. Conclusion CDTs are embracing their new status as an occupational group within dentistry. Core features of becoming a professional group were exhibited including the importance of social and financial status and the need to negotiate their current and future roles in the healthcare system.

Our apologies for any confusion caused by this error. 